\title{
Chemometric Evaluation of Poly(3-hexylthiophene) Electrochemical Aging Path as a Function of Electrochemical Synthesis Variables
}

\author{
Roger Gonçalves ${ }^{a}{ }^{\oplus}$ and Ernesto C. Pereira ${ }^{* a} a$ \\ ${ }^{a}$ Laboratório Interdisciplinar de Eletroquímica e Cerâmica, Departamento de Química, \\ Universidade Federal de São Carlos, CP 676, 13565-905 São Carlos-SP, Brazil
}

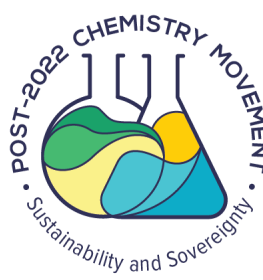

Conductive polymers are versatile materials that can be applied in many different technology fields, especially green energy. They have several advantages, such as easy synthesis, low density, and cost. However, its electric conduction property is also related to material aging with great loss of their characteristics, limiting their applicability. The in-depth study of electrochemical aging can provide useful information for new strategies to overcome this problem. Thus, this work describes the use of factorial design to evaluate the influence of some synthesis variables on the aging of poly(3-hexylthiophene): electrolyte and monomer concentration and water in the synthesis of non-aqueous solution. For this, the properties of the films were evaluated by cyclic voltammetry, electrochemical impedance spectroscopy, and scanning electron microscopy. The accelerated electrochemical aging was performed by applying a potential higher than the superoxidation potential for 20 min steps. It was possible to identify two extreme cases, the conventionally synthesized polymer aged more, while the polymer obtained with the three altered parameters suffered fewer aging effects. It was possible to identify a range of different aging pathways among these. The mechanical stress caused by the several oxidation/reduction cycles of the material aligned to the increasing loss of polymer properties, potentiates aging.

Keywords: poly(3-hexylthiophene), impedance spectroscopy, electrochemical aging, electrochemical degradation, factorial design

\section{Introduction}

Conducting polymers are versatile materials that can be applied in several devices, such as capacitors, ${ }^{1}$ electrochromic displays, ${ }^{2,3}$ and photoelectrochemical cells. ${ }^{4,5}$ As pointed by Kabasakaloglu et al. ${ }^{6}$ their properties are very sensitive to the synthetic parameters. In this sense, it is possible to obtain different materials by adjusting the experimental conditions. However, commercially, organic devices based on conductive polymers have low durability as the main challenger compared to pure inorganic or hybrid devices. It is especially important in those cases where the polymers are the active material and then submitted to successive oxidation/reduction cycles, as in redox devices, such as supercapacitors and batteries. Under this situation, due to their intrinsic conducting properties, there is mass transport to compensate for the charges generated in the polymer chains during the oxidation/reduction processes, which can cause mechanical stress. ${ }^{7,8}$ This is probably one

*e-mail: ernesto@ufscar.br

Editor handled this article: Rodrigo A. A. Muñoz (Associate) of the main aspects which lead to premature aging of the material. ${ }^{9}$

Thus, to build promising commercial devices, it is indispensable to increase the durability of these materials and understand how electrochemical aging occurs. For once, understanding the impacts and how it is caused can lead to a new approach as their lifetime can be increased. Considering these aspects, several studies have been published in the literature involving incorporating inorganic components when the operation of secondary effects, like the self and secondary doping. ${ }^{10-12}$ On the other hand, since premature aging is due to ion intercalation during the redox process, which has mechanical changes, the electrochemically induced aging studies are not sufficiently explored in the literature. However, they can provide useful clues towards understanding and minimizing the damage process.

The conductive polymer family most studied in concerning aging are polyanilines and their derivatives, for which was investigated the dependence of the anion, ${ }^{13,14}$ potential, ${ }^{15,16}$ temperature, ${ }^{17}$ and a kinetic study. ${ }^{18}$ In the case of thiophene derivatives, studies are less extensive. However, it is possible to cite works that evaluated the 
influence of $\mathrm{pK} \mathrm{a}^{19}$ and $\mathrm{pH}^{20}$ of the solvent. It was also noted by Pud, ${ }^{21}$ studying several conducting polymers, that the charge storage characteristic of polypyrrole was not fully lost after electrochemical degradation. In contrast, for other polymers, unique electrochemical features disappeared. Harada et al. ${ }^{22}$ studied the superoxidation region of polythiophenes by cyclic voltammetry. They noted that the process of deactivation is permanent and irreversible for applied potential higher than a crucial value.

Investigating the effects of aging on polythiophenes ${ }^{23}$ is important because they have as main feature the easy addition of substituents groups in the monomers, making them an ideal compound for the synthesis of new polymers to build green energy devices. Also, they have a huge chemically and electrochemically stability in both doped and undoped states. ${ }^{24-27}$ Besides, to improve their processability have been widely proposed the addition of 3-alkyl-substitutes ${ }^{28-31}$ with more than four carbons. ${ }^{32-34}$ Among these, the poly(3-hexylthiophene), P3HT, is the most exploited polythiophene (PTH) derivative once it is soluble in most organic solvents, preserving the inherent properties of other polythiophenes.

Considering these aspects, this work aims to study how the synthesis conditions affect their electrochemical properties and the aging process. We look at the influence of the monomer concentration, electrolyte concentration, and small water addition in the organic media during P3HT electrosynthesis and then during the electrochemical aging process. To this end, electrochemical impedance spectroscopy and electronic scanning microscopy were used. The electrochemical impedance spectroscopy (EIS) data were analyzed using the transmission line models approach. ${ }^{35,36}$ The micrographs were obtained from newly synthesized films because their morphology is strongly connected with electrochemical degradation, as found in previous work. ${ }^{37}$

\section{Experimental}

\section{Factorial design}

The factorial design approach was used to study the effects of three variables: concentration of monomer, electrolyte, and water in organic media. The use of experimental design allows a small number of experiments to verify the impact of the three variables alone and together with side-crossed effects. ${ }^{38,39}$ The experimental design variable values are presented in Table 1 .

It is important to note that (i) $200 \mathrm{ppm}$ of water corresponds to a concentration of $11 \mathrm{mmol} \mathrm{L}^{-1}$, and (ii) $0 \mathrm{ppm}$ means that no water was added to the solvent. The levels studied are represented by the + (plus) sign to the high level and - (minus) to the low level for each variable in the coding table. The nomenclature given to the eight experiments is: letters represent each variable, $\mathrm{m} / \mathrm{M}$ to the concentration of the monomer, e/E the electrolyte and w/W for the water, uppercase or lowercase represent the upper and lower levels, respectively. Then, $\mathrm{mEw}$ is the experiment that used $0.1 \mathrm{~mol} \mathrm{~L}^{-1}$ monomer, $0.2 \mathrm{~mol} \mathrm{~L}^{-1}$ of electrolyte, and $0 \mathrm{ppm}$ of water; while $\mathrm{meW}$ is the experiment that used $0.1 \mathrm{~mol} \mathrm{~L}^{-1}$ monomer, $0.1 \mathrm{~mol} \mathrm{~L}^{-1}$ to $200 \mathrm{ppm}$ of electrolyte and water, respectively; and so on. Each investigation resulted in different P3HT films; all were characterized in triplicate.

Details about the analysis method of the effects on the different responses are described in the Supplementary Information (SI) section.

\section{Materials and methods}

Analytical grade reagents (Sigma-Aldrich, São Paulo, Brazil) were used in this work. The monomer, 3-hexylthiophene was used without any pre-treatment. All solutions were prepared in acetonitrile (ACN) high

Table 1. Table used to planning the factorial design experiments

\begin{tabular}{|c|c|c|c|c|c|c|c|}
\hline \multicolumn{2}{|c|}{ Experiment } & \multicolumn{2}{|c|}{ [Monomer] / $\left(\mathrm{mol} \mathrm{L}^{-1}\right)$} & \multicolumn{2}{|c|}{ [Electrolyte] / $\left(\mathrm{mol} \mathrm{L}^{-1}\right)$} & \multicolumn{2}{|c|}{ [Water] / ppm } \\
\hline No. & Name & Encoded & Real & Encoded & Real & Encoded & Real \\
\hline 1 & mew & -1 & 0.10 & -1 & 0.10 & -1 & $0^{\mathrm{a}}$ \\
\hline 2 & meW & -1 & 0.10 & -1 & 0.10 & +1 & 200 \\
\hline 3 & $\mathrm{mEw}$ & -1 & 0.10 & +1 & 0.20 & -1 & $0^{\mathrm{a}}$ \\
\hline 4 & $\mathrm{mEW}$ & -1 & 0.10 & +1 & 0.20 & +1 & 200 \\
\hline 5 & Mew & +1 & 0.20 & -1 & 0.10 & -1 & $0^{\mathrm{a}}$ \\
\hline 6 & $\mathrm{MeW}$ & +1 & 0.20 & -1 & 0.10 & +1 & 200 \\
\hline 7 & $\mathrm{MEw}$ & +1 & 0.20 & +1 & 0.20 & -1 & $0^{\mathrm{a}}$ \\
\hline 8 & MEW & +1 & 0.20 & +1 & 0.20 & +1 & 200 \\
\hline
\end{tabular}

${ }^{a}$ No water addition into the solution. 
performance liquid chromatography (HPLC) grade (SigmaAldrich, São Paulo, Brazil) and the water used in the experiments was ultra-purified by the Milli-Q system. Eight different solutions were prepared with concentrations listed for anodic electropolymerization of P3HT films following the table factorial design. On the other hand, the aging experiment was realized on a blank solution of $0.1 \mathrm{~mol} \mathrm{~L}^{-1}$ $\mathrm{LiClO}_{4}$ in anhydrous acetonitrile. The electropolymerization has been carried out potentiostatically $(1.5 \mathrm{~V} v s . \mathrm{Ag})$ on a platinum electrode (area $=0.2 \mathrm{~cm}^{2}$ ) up to a growing charge of $1.0 \mathrm{C} \mathrm{cm}^{-2}$ per mole of monomer in the synthesis. As the auxiliary electrode, a platinum net was used. All experiments were conducted at $298 \mathrm{~K}$ in a dry chamber with an $\mathrm{N}_{2}$ atmosphere to avoid external influences over the observed results. The electrosynthesis and electrochemical characterizations were carried using an Autolab $^{\odot}$ potentiostat/ galvanostat model PGSTAT302N with FRA 3.0M module (Metrohm-Autolab, Utrecht, Netherlands).

\section{Characterization and electrochemical aging}

All the characterization and the electrochemical aging were performed using $0.1 \mathrm{~mol} \mathrm{~L}^{-1} \mathrm{LiClO}_{4}$ as supporting electrolyte. The cyclic voltammograms (CV) were recorded in the potential range of 0.0 up to $1.0 \mathrm{~V}$ versus $\mathrm{Ag}$ at ten $\mathrm{mV} \mathrm{s}^{-1}$. The impedance spectra were obtained at a $0.9 \mathrm{~V}$ (versus $\mathrm{Ag}$ ) DC potential before each degradation step. The investigated frequency window was between $10 \mathrm{kHz}$ and $10 \mathrm{mHz}$, collected 10 points per decade with $10 \mathrm{mV}$ of alternating voltage amplitude. Before acquiring each impedance spectra and voltammograms, the electrode was polarized for $180 \mathrm{~min}$ at $0.9 \mathrm{~V}$ potential to reach the steady-state.

The degradation of the films was performed by polarizing the electrode at a potential $1.10 \mathrm{~V}$ for $20 \mathrm{~min}$

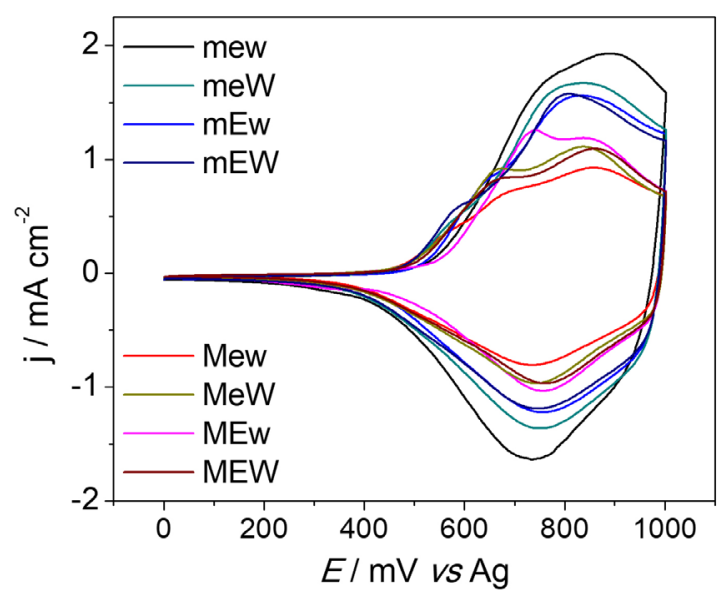

and then carried out for three voltammetric cycles at the same potential window described above at a sweep rate of $50 \mathrm{mV} \mathrm{s}^{-1}$. Here, it is important to describe that it is common to use a narrow potential window during the electrochemical characterization of a conducting polymer compared to the potential value used during the electropolymerization under potentiostatic conditions. The applied potential for oxidizing a monomer molecule is higher than those required to oxidize a dimer and continuously decreases as the polymeric chain increases ${ }^{40-42}$ Indeed, to investigate the electrochemical properties of the polymer in a blank (i.e., in the absence of monomer) if the polymerization potential was used as the anodic vertex in the cyclic voltammetry, the material will be completely degraded in only one cycle. Then, we choose to age the polymer using a potential value that leads to slow enough degradation to be followed using an adequate number of experiments.

The scanning electron microscopy (SEM) characterization was performed using a FEI Inspect S 50, with a electron beam of $5 \mathrm{keV}$.

\section{Results and Discussion}

\section{Effect of variables on the "pristine polymer" properties}

The first moment will discuss the properties of the new synthesized films data to enable further discussion based on how these results relate to the observed changes and the electrochemical aging. Thus, Figures $1 \mathrm{a}$ and $1 \mathrm{~b}$ show the voltammetric profile and impedance spectra, normalized by each film's solution resistance (Rs) immediately after the electrodeposition. In the voltammetric profile, it is possible to observe that the samples obtained from a $0.2 \mathrm{~mol} \mathrm{~L}^{-1}$ of monomer solution (M-- notation, which means high monomer concentration for any other variable conditions)

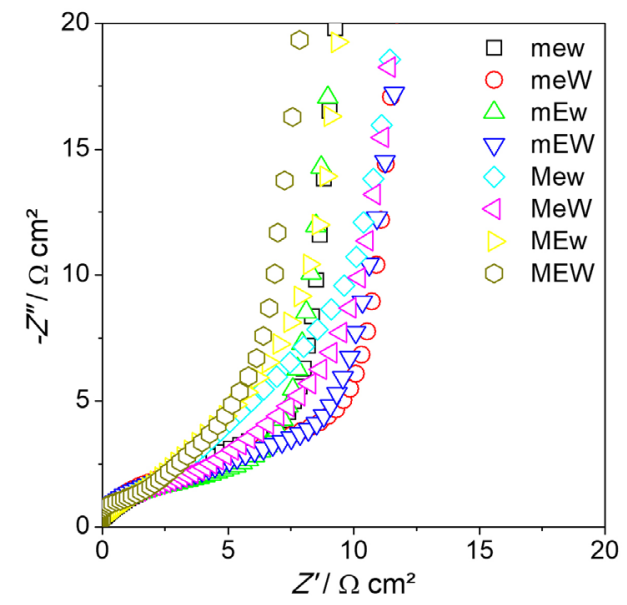

Figure 1. Cyclic voltammograms and Nyquist plots (normalized by solution resistance) for the P3HT films. CV speed $=50 \mathrm{mV} \mathrm{s} \mathrm{s}^{-1}$. EIS frequency range from $10 \mathrm{kHz}$ down to $10 \mathrm{mHz}$ at $0.9 \mathrm{~V}$, AC voltage of $10 \mathrm{mV}$. 
present lower current density, below $1.25 \mathrm{~mA} \mathrm{~cm}^{-2}$ and more evident twofold peak. The EIS data also showed many differences in each of the syntheses. However, regardless of this fact, all the spectra obtained still match the expected for porous electrodes. Thus, they were adjusted according to the transmission line model already proposed in previous papers ${ }^{37,42,43}$ and described in the Supplementary Information section. The quality and reproducibility of the adjustments were also observed, with a coefficient of ratio greater than 0.9996 and $\chi^{2}$ (sum of square errors) below $1 \times 10^{-4}$ for all adjustments.

As previously stated, because there is more than one oxidation process for some samples, the current density was not chosen as a response in the analysis, instead was used the anodic and cathodic charge from voltammetry. Figure 2 presents Pareto's representation of the variable's effects from voltammetric and EIS data. It is possible to observe, in Figure 2, that the charged relationship is not affected by the variables in the studied levels, because the values of the effects are below the error range. Therefore, the coulomb efficiency redox process is independent of these synthesis variables. It means that both anodic and cathodic charges are affected in the same way. Once the value of the total anodic charge gives a clear idea of the extent of the effects of degradation on the material, because it represents not only the change in the electroactive area of the film through the area of the voltammogram but also the partial loss of its electrochemical properties, the anodic charge will be chosen for the analysis of the variable effects. Thus, it was verified that increasing the monomer concentration decreases the charge calculated voltammogram area while increasing electrolyte and water concentration affects increasing this parameter.

In turn, Figures $2 \mathrm{c}$ and $2 \mathrm{~d}$ show the Pareto's plot for the charge transfer resistance and the double layer capacitance, respectively. The effects over both are almost the same; the increase in the concentration of monomer or the electrolyte decreases the charge transfer resistance
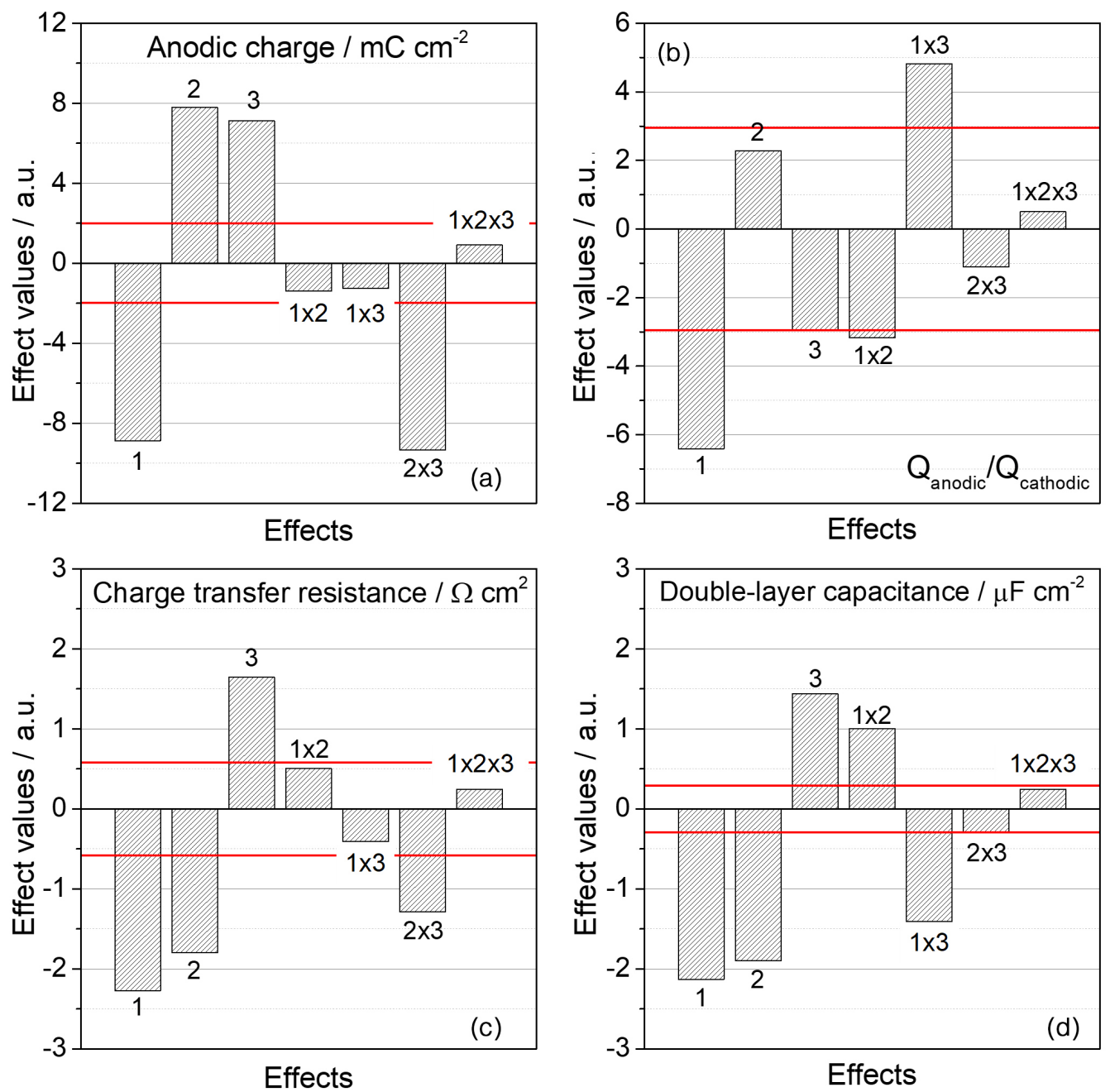

Figure 2. Pareto's plot of the effect's values for each variable over the responses: (a) anodic charge, (b) anodic/cathodic charge relation, (c) charge transfer resistance, and (d) double-layer capacitance. The red line indicates the error of the calculated effects. 
and the double-layer capacitance; nevertheless, increasing both leads to an increase of these parameters. As stated before, the synthesis with a more monomer concentrated solution leads to a more compact structure with fewer pores. Also, the pores could become narrower and shallower. It means a less electroactive area, which affects the doublelayer capacitance value. On the other hand, using more electrolytes during the synthesis leads to a better charge distribution, resulting in a more homogeneous polymer surface. This homogeneity guarantees a surface that better transfers charge (less resistance) and presents fewer irregularities and electroactive areas.

To better understand the changes in the properties of the newly synthesized films, the morphology was characterized through scanning electron microscopy (SEM) images, shown in Figure 3. From the first line to the second, it is possible to observe the monomer concentration increase effect. While, from the first column to the second and third, the effects of water and electrolyte concentration are observable, respectively. Finally, from the first column to the last, the effect of increasing both water and electrolyte could be evaluated. The more pronounced difference can be appreciated by increasing the amount of monomer, which causes a drastic change in the morphology of the films.

Similarly, these changes observed in the morphology follow the propositions raised before, i.e., more/less dense morphology. A solution with a higher monomer concentration is expected to favor the formation of shortlength chains,${ }^{44,45}$ which causes the formation of a denser/ less porous structure. The increase in the electrolyte concentration, as can be observed, promotes the formation of a more homogeneous morphology. This characteristic is clearer when Mew and MEw samples were compared. It is stated that a higher concentration of electrolyte allows for greater homogeneity in the distribution of current lines, making the deposit also become more homogeneous; in addition, the ohmic drop suffered in the vicinity of the electrode is minimized, allowing the activation reaction of the monomer can occur further away from the electrode surface, where there is a higher concentration of other species for the continuation reaction to proceed. These two factors contribute to the film-forming more homogeneously and with a predominance of longer polymeric chains, which contributes to better electrode surface coverage.

\section{Effect of synthesis variables in electrochemical aging}

Once, as observed in previous work, ${ }^{37}$ there is a great difference between the samples. Thus, the relative percentage change was used to compare the influence of these parameters over the aging process. Therefore, this section will discuss how the synthesis parameters affect the aging process of the samples. Besides, it was also observed that there are two regimes of property changes; the rates of change before and after $100 \mathrm{~min}$ are different. This fact can be observed in Figure $4 \mathrm{a}$, representing the percentage of anodic charge in the function of aging time. However, a more in-depth discussion of these rates will be made later. Besides, Figures $4 \mathrm{~b}$ and $4 \mathrm{c}$ show de cyclic voltammetry after $100 \mathrm{~min}$ and after $180 \mathrm{~min}$ of electrochemical aging. In general, before $100 \mathrm{~min}$, all the voltammetric profiles remain remarkably similar to before the aging time, with a change in the anodic charge values, as expected. The voltammograms that least suffered changes correspond to those less aged samples. On the other hand, after $180 \mathrm{~min}$, it is interesting to observe that all the samples obtained with

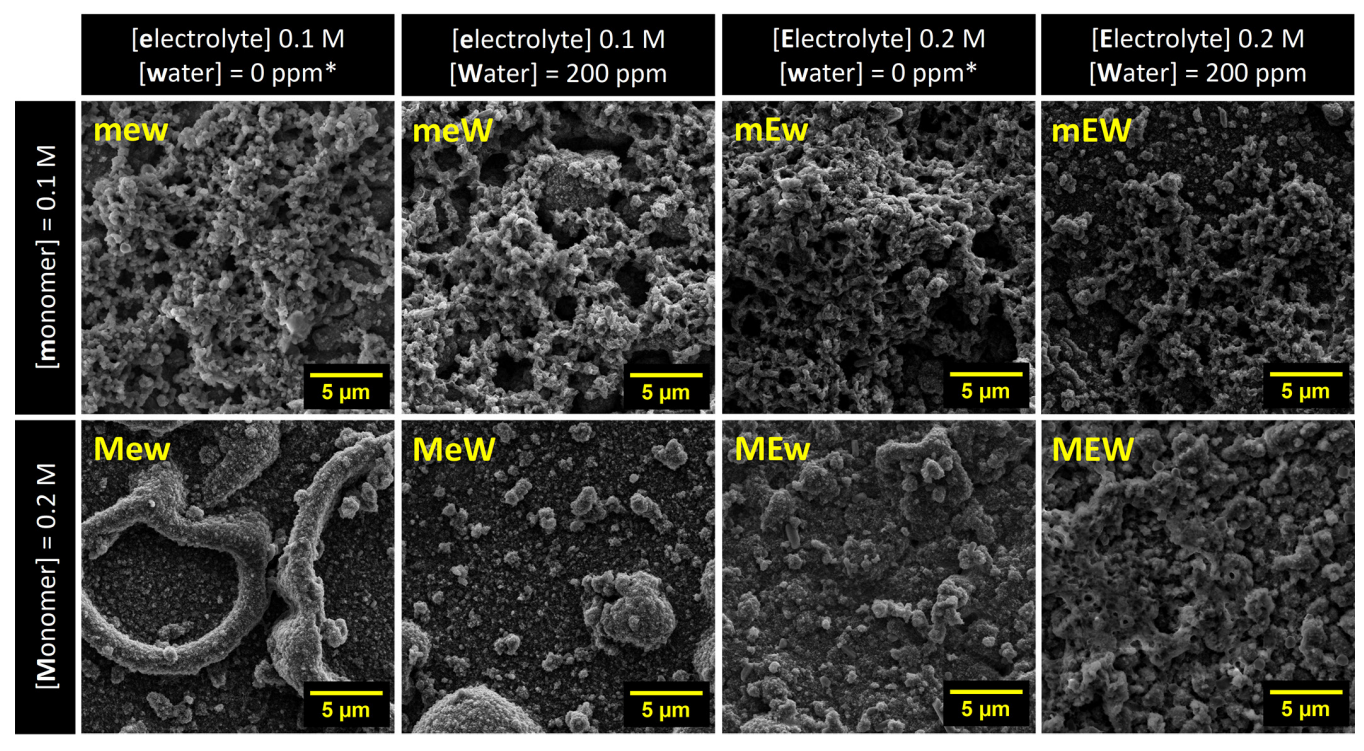

Figure 3. Scanning electron microscopy micrographs of the fresh samples. Obtained in 10k $\times$ magnification. 
a lower concentration of monomer aged much more than the others. The most evident change that can be observed is the appearance of a second oxidation peak, which is already discussed in the literature ${ }^{19-21}$ and associated with stepwise oxidation of polymeric chains with distinct properties, which reinforces the discussion about two aging regimes, before and after $100 \mathrm{~min}$ of aging.

Figure 5 a shows the rate of polymer resistance increase, the rate I was obtained from time 0 to $100 \mathrm{~min}$ and rate II from time 100 to $180 \mathrm{~min}$. It is noticeable that both rates exhibit quite different values for most samples. Besides, rate II is higher than rate I, mainly for more samples. Figures $5 \mathrm{~b}$ and $5 \mathrm{~b}$ show Pareto's plot of the effects over the rate I and rate II; interestingly, each one is affected by different variables. This observation could give an idea about the aging mechanisms. These effects will be interpreted considering the variables' influence over the polymer properties, as discussed before. Therefore, the polymer resistance rate that grows at the first moment of the degradation depends on the pore size; as less they are, the faster the rate, the smaller the monomer amount used in synthesis. The ions trapped in the polymer structure will significantly impact polymer strength at the second degradation regime. This fact may be related to the mechanical stress that the polymer undergoes with the ion's intercalation/deintercalation. Due to the pores narrowing, this process forces the structure of the polymer more strongly so that the higher the number of ions already present inside the structure, the less stress the polymer will suffer and the lower the degradation rate.

Figure 6 shows interesting impedance parameters as a function of the aging time. The relative electrode distance was obtained applying the relation presented in Figure 6b. For this relationship to be used in this way, it was assumed that: (i) the resistivity of the medium present between the working electrode (WE) and the reference electrode (RE) did not change significantly over the aging process, and (ii) the electrode area changed much less than the change experienced by the polymer volume. It is noticeable that the solution resistance follows the same behavior as the other parameters. The one that most aged is the one that exhibits the higher variation, and the contrary is also a truth.
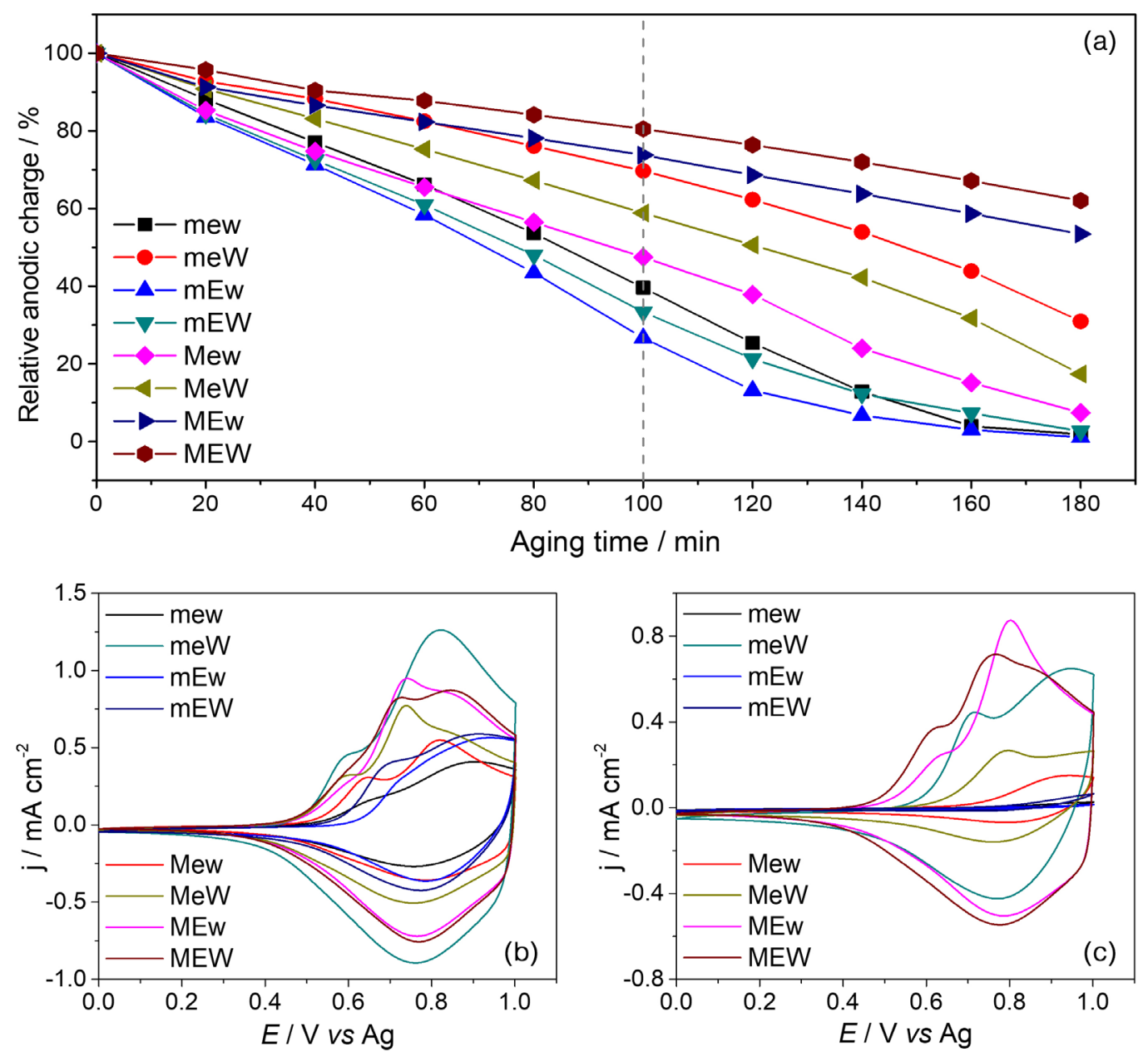

Figure 4. (a) Relative anodic charge in the function of the aging time and cyclic voltammograms of the aged samples (b) before $100 \mathrm{~min}$ and (c) after $180 \mathrm{~min}$. 

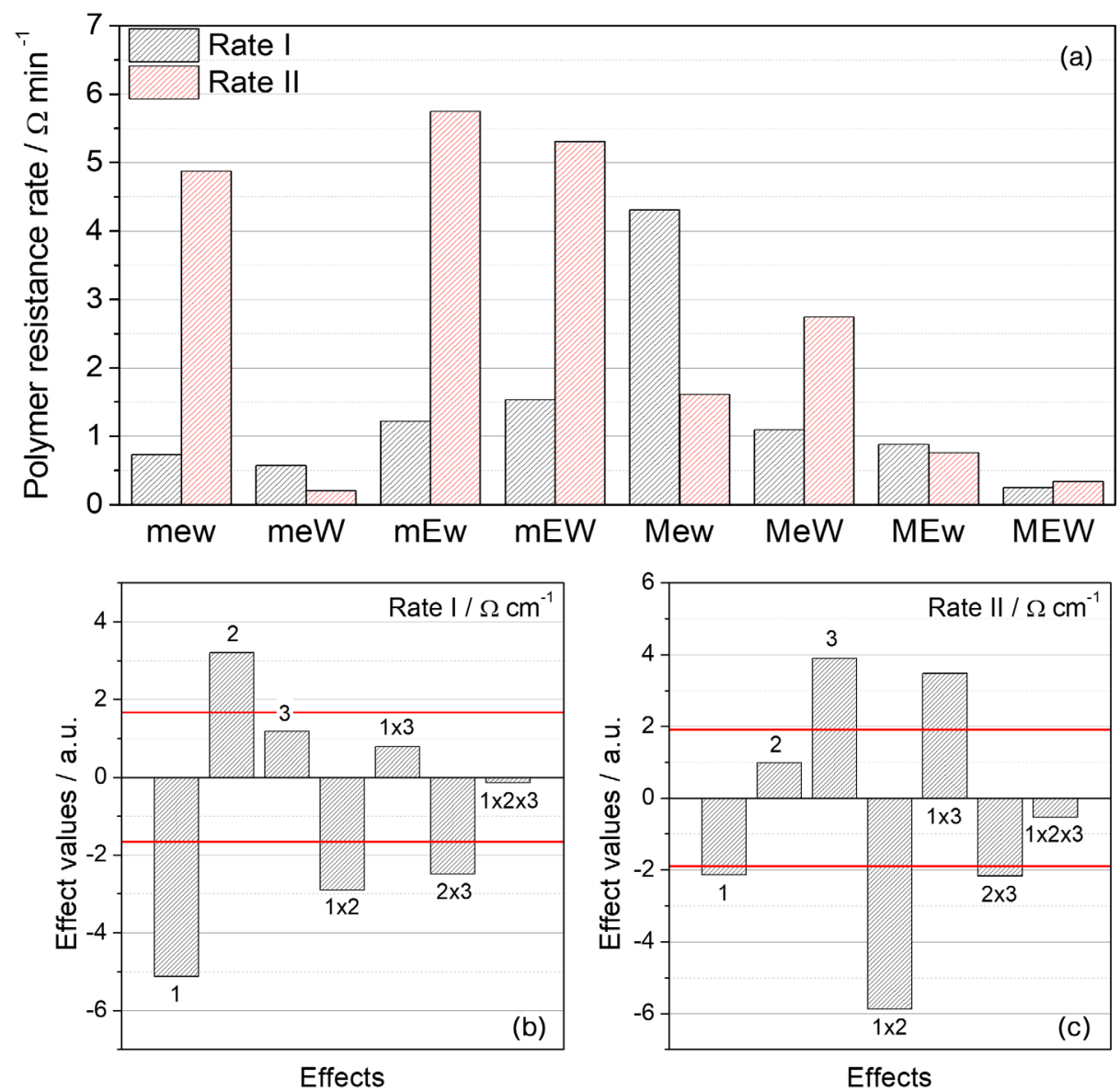

Figure 5. (a) Rate of polymer resistance change for the samples. Pareto's plot of the values of the effects for each variable over the responses: (b) rate I and (c) rate II.

On the other hand, there are no two degradation regimes, an almost linear increase for all samples. This fact may mean that aging happens in a single step from a morphological point of view. In contrast, from an electronic point of view, it does not occur at the first moment, or it occurs at very low rates, with an exponential increase after a certain degradation time (100 min). Hence, degradation occurs faster due to the combined effect of these two behaviors.

Additionally, comparing Figures $6 \mathrm{c}$ and $6 \mathrm{~d}$ is possible to observe that the two main resistances found in the system behave differently. Since the resistance value evolution for each sample is quite different, the comparison was made with the normalized data, i.e., they all start at the same value, in this case, $1.0 \Omega$. The physical significance of these two resistances, described in the design of the impedance data adjustment model, allows one to evaluate the electrochemical properties of the material.

It is possible to observe that the behavior of both resistances in the function of the aging time is different. Each sample suffered a different aging path, and as a consequence, each sample showed different responses. Thus, to view how each sample ages, Figures 6e and 6f present the $R_{c t}$ versus $R_{p o l}$ plots. It is important to reinforce that $R_{\mathrm{ct}}$ refers to the aging of the electrochemical response, the electronic structure of the polymer changes, and the $\mathrm{R}_{\mathrm{pol}}$ to the 'physical' aging, i.e., the polymer chain length decreases. Although the data is not linear enough for regression to be made, tentative lines were drawn for the analysis to be performed. Because of the similarity of behaviors, Figure 6e shows the samples obtained with less monomer quantity; it is observed that, except for the meW sample, which follows a linear increase, the other samples present two patterns of increase, as already seen in other properties, before $100 \mathrm{~min}$ and after. The first region of $\mathrm{mEw}$ and $\mathrm{mEW}$ and the meW showed a slope close to $45^{\circ}$, which means that the 'physical' and electrochemical aging occur.

On the other hand, the first region of the mew sample presents a higher slope, meaning that, in this region, the 'physical' aging is more pronounced than the electrochemical. For the second region, aging also changes from one region to another once the slope changes. Thus, $\mathrm{mEw}$ and mEW suffer a more 'physical' aging while the mew suffers a majority electrochemical aging. 

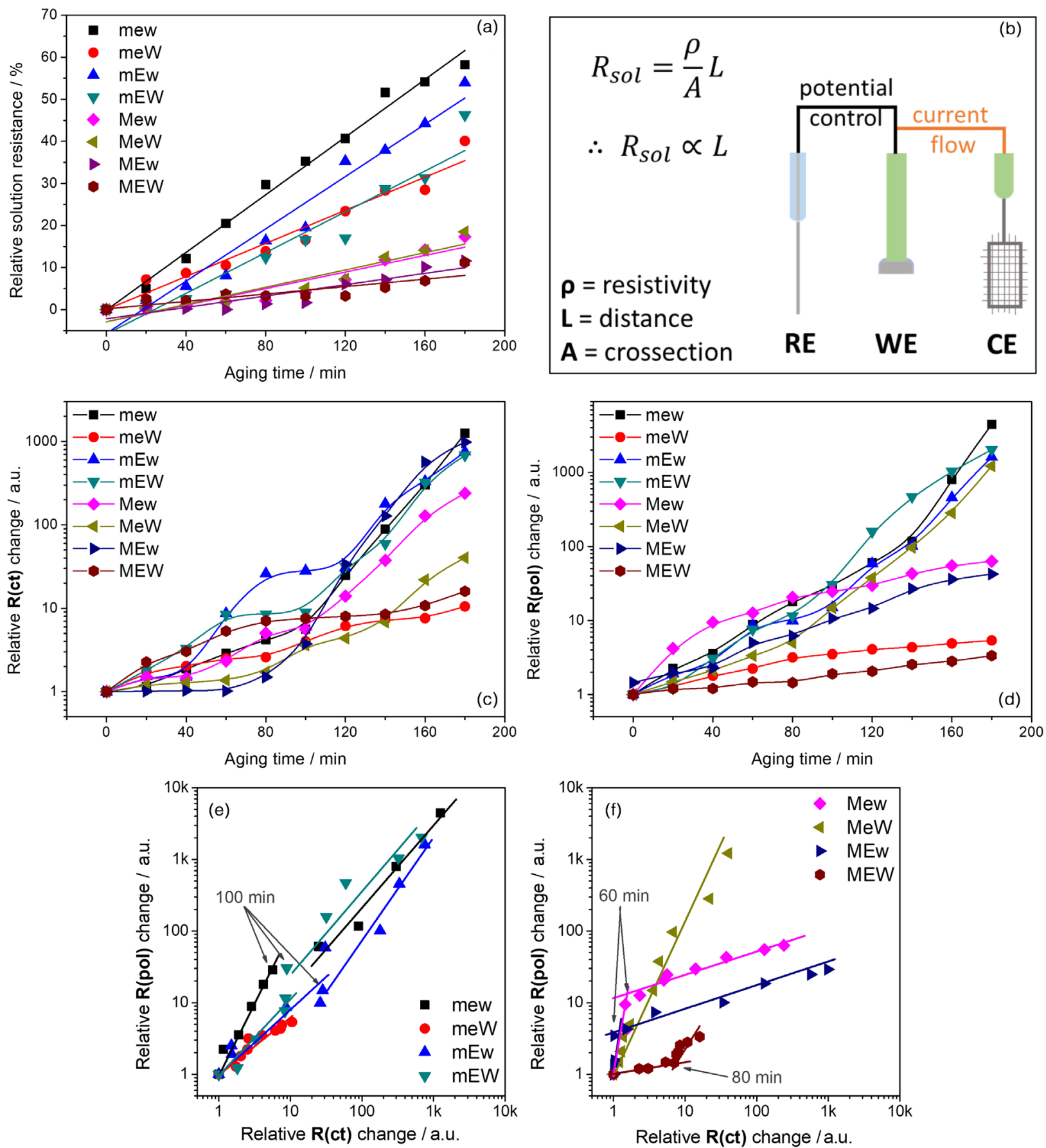

Figure 6. (a) Relative distance between the working electrode and the reference electrode, (b) schematic representation of the electrochemical cell and the formula used to calculate the relative distance, (c) the relative charge transfer resistance, (d) relative polymer resistance in the function of the aging time and the relative $\mathrm{R}_{\mathrm{ct}}$ and $\mathrm{R}_{\mathrm{pol}}$ plots for (e) lower and (f) higher monomer concentration.

The samples synthesized from the solution with a higher monomer concentration are in Figure 6f. A quite different negation can be observed for this set of samples; first, the MeW sample presents a linear behavior with an extremely high slope, more 'physical' aging. In the second place, it is noticeable that the behavior of the Mew and Mew samples are similar, a first region (until $60 \mathrm{~min}$ ) with a high slope and a second one with an exceptionally low slope. These samples are two of those that aged little throughout the test. Possibly it is because there is an early change in the aging profile and that in the second stage, this aging occurs very slowly. Finally, the sample that aged the least, MEW, also has a two-step behavior, the first with a small slope and, after $80 \mathrm{~min}$, the second stage with a slope close to $45^{\circ}$.

It is important to note that it is impossible to separate one aging from another, i.e., electrochemical and 'physical' 
aging occur together. Still, it is interesting to make this analysis based on the two resistances. It provides an idea of how the aging process unfolds-electrochemical and the evidence that each sample undergoes a different way. Therefore, in general, the samples that lost most properties with the overoxidation process did not have major differences between the resistance increase regimes in the $\mathrm{R}_{\mathrm{ct}}$ versus $\mathrm{R}_{\mathrm{pol}}$ graph; for example, the linearity of this graph is directly proportional to the decrease of properties. In this sense, it can be concluded that the action of both aging types causes a much more severe properties loss under the conditions studied.

For the discussion, physical effect was separate from electrochemical effect because, during the process of oxidation and reduction of the polymer, it undergoes a structural transformation (from the aromatic to the quinoid form), which causes a conformational rearrangement in the polymer chains and which, in a macro view affects the structure of the whole film. Thus, our mention of physical aging is the mechanical aging of the film in the face of the various reduction/oxidation cycles that the polymer chain undergoes. Thus, since physical rearrangement is necessary for oxidation/reduction, once re-conformation is no longer possible, locally, the polymer will no longer exhibit conduction, which can affect large portions of the polymer film, leading to a large loss of properties, as seen in the mew sample. On the other hand, we believe that electrochemical aging affects only small portions of the polymeric chain and, therefore, exhibits less loss of properties or a slower loss, as it is an aging process that occurs little by little, as seen in the MEW sample, especially under the conditions studied.

\section{Conclusions}

The factorial design is a powerful tool to define a limited set of experiments that allows verifying the influence of selected variables over a whole system. The influence of the monomer and electrolyte concentration, besides the presence of water in the non-aqueous solution of synthesis, was evaluated. The first two parameters were chosen because they are commonly changed from a work to another. On the other hand, some papers state that the presence of water during the synthesis of poly(3hexylthiophene) leads to the formation of the worst quality film in terms of properties. ${ }^{46,47}$ Then, with only eight experiments, it was possible to study the influence of the selected variables and the cross effects. Using the electrochemical impedance data, it was possible to notice a series of behaviors of each of the samples, which were strongly influenced by the studied parameters.
In a previous work, ${ }^{37}$ the aging profile of two samples was presented, which are the stems of the entire sample set. It was possible to identify a range of paths that aging can follow among these samples. One important thing observed was that the mechanical stress caused by the material's various oxidation and reduction cycles, together with the increasing loss of polymer properties, potentiates aging. Finding that aging may not necessarily occur linearly and may have different aging regimes is quite interesting in outlining strategies so that the overall problem, the low electrochemical durability of the polymer, can be effectively remedied. Besides, as stated before, the samples that exhibited the worst initial properties also presented less aged (weakened) parameter values.

\section{Supplementary Information}

Supplementary data are available free of charge at http://jbcs.sbq.org.br as PDF file.

\section{Acknowledgments}

The authors acknowledge the financial support provided by the Brazilian funding agencies, which include the Brazilian National Council for Scientific and Technological Development - CNPq (grant No. 465571/2014-0), São Paulo Research Foundation (2011/10897-2, 2013/07296-2, 2015/17141-1 and 2017/11986-5, 2017/06536-0), FAPESP-GSK (2014/50249-8), and the Coordenação de Aperfeiçoamento de Pessoal de Nível Superior (CAPES - Finance Code 001).

\section{References}

1. Laforgue, A.; Simon, P.; Sarrazin, C.; Fauvarque, J.-F.; J. Power Sources 1999, 80, 142.

2. Nicho, M.; Sol. Energy Mater. Sol. Cells 2004, 82, 105.

3. Varis, S.; Ak, M.; Tanyeli, C.; Akhmedov, I. M.; Toppare, L.; Eur. Polym. J. 2006, 42, 2352.

4. Guerrero, A.; Boix, P. P.; Marchesi, L. F.; Ripolles-Sanchis, T.; Pereira, E. C.; Garcia-Belmonte, G.; Sol. Energy Mater. Sol. Cells 2012, 100, 185

5. Boix, P. P.; Ajuria, J.; Etxebarria, I.; Pacios, R.; GarciaBelmonte, G.; Thin Solid Films 2012, 520, 2265.

6. Kabasakaloglu, M.; Kiyak, T.; Toprak, H.; Aksu, M. L.; Appl. Surf. Sci. 1999, 152, 115.

7. Anquetil, P. A.; Yu, H.; Madden, J. D.; Swager, T. M.; Hunter, I. W. In Smart Structures and Materials; Bar-Cohen, Y., ed.; International Society for Optics and Photonics: California, United States, 2003, p. 42. 
8. Westover, A. S.; Shabab, F. N.; Tian, J. W.; Bernath, S.; Oakes, L.; Erwin, W. R.; Carter, R.; Bardhan, R.; Pint, C. L.; J. Electrochem. Soc. 2014, 161, E112.

9. Marmisollé, W. A.; Inés Florit, M.; Posadas, D.; J. Electroanal. Chem. 2012, 673, 65.

10. Nguyen, T.-P.; Surf. Coat. Technol. 2011, 206, 742.

11. Malinauskas, A.; J. Power Sources 2004, 126, 214.

12. Christinelli, W. Ap.; Gonçalves, R.; Pereira, E. C.; J. Power Sources 2016, 303, 73.

13. Tang, H.; Kitani, A.; Shiotani, M.; Electrochim. Acta 1996, 41, 1561.

14. Zhang, A. Q.; Cui, C. Q.; Lee, J. Y.; Synth. Met. 1995, 72, 217.

15. Arsov, L. D.; Plieth, W.; Koßmehl, G.; J. Solid State Electrochem. 1998, 2, 355.

16. Marmisollé, W. A.; Florit, M. I.; Posadas, D.; J. Electroanal. Chem. 2012, 669, 42.

17. Tang, H.; Ding, Y.; Zang, C.; Gu, J.; Shen, Q.; Kan, J.; Int. J. Electrochem. Sci. 2014, 9, 7239.

18. Malinauskas, A.; Maz, R.; Mažeikienè, R.; Malinauskas, A.; Maz, R.; Mažeikienè, R.; Malinauskas, A.; Synth. Met. 2001, 123, 349.

19. Wang, J.; Electrochim. Acta 1997, 42, 2545.

20. Tourillon, G.; J. Electrochem. Soc. 1983, 130, 2042.

21. Pud, A. A.; Synth. Met. 1994, 66, 1.

22. Harada, H.; Fuchigami, T.; Nonaka, T.; J. Electroanal. Chem. Interfacial Electrochem. 1991, 303, 139.

23. Kim, T. H.; Choi, K. I.; Kim, H.; Oh, S. H.; Koo, J.; Nah, Y. C.; ACS Appl. Mater. Interfaces 2017, 9, 20223.

24. Kaneto, K.; Yoshino, K.; Inuishi, Y.; Solid State Commun. 1983, 46, 389.

25. Waltman, R. J.; Bargon, J.; Diaz, A. F.; J. Phys. Chem. 1983, $87,1459$.

26. Kaneto, K.; Yoshino, K.; Inuishi, Y.; Jpn. J. Appl. Phys. 1983, 22, L412.

27. Leclerc, M.; Faid, K.; Adv. Mater. 1997, 9, 1087.

28. van de Leur, R. H. M.; de Ruiter, B.; Synth. Met. 1991, 44, 327.
29. van de Leur, R. H. M.; de Ruiter, B.; Breen, J.; Synth. Met. 1993, 54, 203.

30. van de Leur, R. H. M.; Synth. Met. 1992, 51, 69.

31. Binh, N. T.; Gailberger, M.; Bässler, H.; Synth. Met. 1992, 47, 77.

32. McCullough, R. D.; Tristram-Nagle, S.; Williams, S. P.; Lowe, R. D.; Jayaraman, M.; J. Am. Chem. Soc. 1993, 115, 4910.

33. Yoshino, K.; Morita, S.; Uchida, M.; Muro, K.; Kawai, T.; Ohmori, Y.; Synth. Met. 1993, 55, 28.

34. Yang, C.; Orfino, F. P.; Holdcroft, S.; Macromolecules 1996, 29,6510 .

35. De Levie, R.; Electrochim. Acta 1965, 10, 113.

36. Buck, R. P.; Mundt, C.; Electrochim. Acta 1999, 44, 1999.

37. Gonçalves, R.; Correa, A. A.; Pereira, R.; Pereira, E. C.; Electrochim. Acta 2016, 190, 329.

38. Terezo, A. J.; Pereira, E. C.; Electrochim. Acta 2000, 45, 4351.

39. Ronconi, C. M.; Pereira, E. C.; J. Appl. Electrochem. 2001, 8, 319.

40. del Valle, M. A.; Gacitúa, M. a.; Canales, L. I.; Díaz, F. R.; J. Chil. Chem. Soc. 2009, 54, 260.

41. Vorotyntsev, M. A.; Zinovyeva, V. A.; Konev, D. V. In Electropolymerization: Concepts, Materials and Applications; Cosnier, S.; Karyakin, A. A., eds.; Wiley VCH: Weinheim, 2010, p. 27-50.

42. Correa, A. A.; Gonçalves, R.; Pereira, R.; Pereira, E. C.; J. Appl. Polym. Sci. 2017, 134, 44368.

43. Gonçalves, R.; Paiva, R. S.; Pereira, E. C.; J. Solid State Electrochem. 2021, 25, 1567.

44. Waltman, R. J.; Bargon, J.; Can. J. Chem. 1986, 64, 76.

45. Sabouraud, G.; Sadki, S.; Brodie, N.; Chem. Soc. Rev. 2000, 29, 283.

46. Das, S.; Chatterjee, D. P.; Ghosh, R.; Nandi, A. K.; RSC Adv. 2015, 5, 20160.

47. Zhou, M.; Heinze, J.; J. Phys. Chem. B 1999, 103, 8451.

Submitted: September 30, 2021 Published online: March 4, 2022 